\title{
A PARABOLIC SYSTEM MODELING THE THERMOELASTIC CONTACT OF TWO RODS
}

\author{
BY \\ KEVIN T. ANDREWS, PETER SHI, MEIR SHILLOR, AND STEVE WRIGHT \\ Oakland University, Rochester, Michigan
}

\begin{abstract}
We consider an initial-boundary value problem for a nonlinear parabolic system that arises naturally in modeling behavior of two (possibly dissimilar) thin homogeneous rods. Each rod is held fixed at one end and is free to expand or contract at the other, as a result of the evolution of its temperature and stress fields. The two rods may also come into contact at their free ends. We establish the existence and uniqueness of a strong solution to the system, assuming that the thermal expansion coefficients are small. The proofs rely on a priori estimates, the functional method of Ladyzhenskaya, and Schauder's fixed-point theorem.
\end{abstract}

1. Introduction. Problems involving thermoelastic contact arise naturally in many applied situations, particularly those involving industrial processes where two or more materials may come into contact or may lose contact as a result of thermoelastic expansion or contraction. The purpose of this paper is to model one such situation: the mechanical behavior of two dissimilar rods which are each fixed at one end but which may come into contact at their free ends as a result of thermoelastic expansion. Our model for the mechanical behavior will consist of a system of energy and elasticity equations involving the temperature and displacement of each rod. We will operate within the framework of linear thermoelasticity and treat the process as quasistatic. Under these assumptions we can show that the problem can be reformulated and reduced to solving an initial-boundary value problem for a nonlinear parabolic system containing only the two temperatures. By contrast the general $n$-dimensional problem of thermoelastic contact considered in [17] does not appear to admit such a reformulation. The parabolic system treated in the present paper contains source terms that are nonlocal as well as nonlinear; moreover, if the problem were set as a variational inequality the associated operator would be nonmonotone and noncoercive, so we could not apply standard results to obtain a solution. Nevertheless, we can show that under appropriate assumptions on the data this system has a unique

Received August 3, 1992.

1991 Mathematics Subject Classification. Primary 35K50; Secondary 73C35, 73T05.

Key words and phrases. Thermoelastic contact, parabolic system, Schauder's fixed-point theorem, Signorini's condition, functional method.

(C)1995 Brown University 
strong solution, i.e., a solution where each temperature lies in a Sobolev space of the form $W_{2}^{2,1}\left(\Omega_{T}\right)$.

This particular two-rod problem has not been modeled previously. In fact, to our knowledge, the only existing paper dealing with this exact situation is by Barber and Zhang [5], who use linear stability analysis and numerical simulations to examine the transient behavior. However, similar problems with simpler geometric settings have been treated in both the engineering literature $[4,19]$ and the mathematical literature $[1,2,3,4,10,14,15,16,18,20,21]$. These papers contain a variety of linear and nonlinear boundary conditions, but in each case the problem involves but a single temperature and a single displacement so that reformulation leads to one nonlinear equation for a single temperature. The present paper deals with a more complicated situation: two temperatures that are coupled not only by a nonlinear system of equations but also in the boundary conditions. The system is also unusual in that each temperature function is defined on a different domain. At the fixed ends of the rods we prescribe Dirichlet boundary conditions but at the free ends we assume that heat exchange occurs. In modeling this heat exchange we will suppose that the heat conduction coefficients and the heat exchange coefficient are all constants. Consequently, the coupled boundary conditions depend linearly on the two temperatures. The case in which the heat exchange coefficient is not constant but is permitted to vary with the temperature as in $[1,2,3]$ we leave for another study. However, we remark that numerical simulations involving this more general boundary condition have been carried out in [7].

We now describe the remaining sections of this paper. Section 2 contains a complete description of the model and describes informally how the problem can be reformulated in terms of the temperatures only. Section 3 gives a precise statement of the resulting parabolic problem and of the existence and uniqueness results. The proofs of existence and uniqueness are given in Sec. 4. The proof of existence combines the functional method of Ladyzhenskaya [11] with an application of Schauder's fixed point theorem. This method of proof is in contrast to the methods employed in the proofs of existence results for the single parabolic equations considered in [2] and [3], which use estimates derived in part from the maximum principle. This latter tool is not available to us here since we are dealing with a parabolic system rather than a single equation.

2. The model. In this section we derive a model for the evolution of a system of two (possibly) dissimilar rods that may come into contact as a result of thermoelastic expansion.

The physical setting consists of two thin rods, each of which is clamped at one end but which may come into contact at their free ends. We assume that the process is independent of all but the horizontal variable so that we can describe the reference configuration of the left rod as $0 \leq x \leq l_{1}$ and of the right rod as $l_{2} \leq x \leq 1$, both in nondimensional units. The fixed ends occur at $x=0$ and $x=1$ while the ends $x=l_{1}$ and $x=l_{2}$ are free to expand or contract. Obviously $l_{1}$ and $l_{2}$ must satisfy $0<l_{1} \leq l_{2}<1$. We let $g=l_{2}-l_{1}$ denote the gap between the rods in the reference configuration. The physical setting is given in Figure 1. 


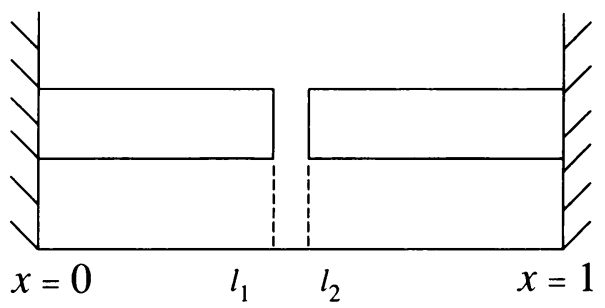

FIG. 1. The setting

We let $\theta_{1}=\theta_{1}(x, t)$ and $\theta_{2}=\theta_{2}(x, t)$ denote the temperatures of the rods, and let $u_{1}=u_{1}(x, t)$ and $u_{2}=u_{2}(x, t)$ be their displacements. Thus $\theta_{1}$ and $u_{1}$ are defined on $0 \leq x \leq l_{1}$ while $\theta_{2}$ and $u_{2}$ are defined on $l_{2} \leq x \leq 1$. In all four cases $0<t<T$. The reference configurations are chosen as the configurations that the rods would have with both $u_{i}$ and $\theta_{i}$ set equal to zero, for $i=1,2$.

We assume that the process is quasistatic, i.e., that the accelerations $u_{1 t t}$ and $u_{2 t t}$ are negligible. Consequently, the system of energy and elasticity equations takes the following form (see, e.g., [15] or [2]):

$$
\begin{aligned}
\theta_{1 t}-\theta_{1 x x} & =-a_{1} u_{1 x t} & & \text { in } \Omega_{1 T}, \\
u_{1 x x} & =a_{1} \theta_{1 x} & & \text { in } \Omega_{1 T}, \\
d \theta_{2 t}-\theta_{2 x x} & =-a_{2} u_{2 x t} & & \text { in } \Omega_{2 T}, \\
u_{2 x x} & =a_{2} \theta_{2 x} & & \text { in } \Omega_{2 T},
\end{aligned}
$$

where $\Omega_{1 T}=\left\{0<x<l_{1}, 0<t \leq T\right\}$ and $\Omega_{2 T}=\left\{l_{2}<x<1,0<t \leq T\right\}$ for $T>0$. Here $a_{1}, a_{2}$, and $d$ are positive constants related to material properties of the rods. In deriving the system of equations we have also assumed that there are no external applied forces or interior heat sources present. We make this assumption merely to simplify our calculations, as the inclusion of terms to take these matters into account poses no additional mathematical difficulties.

Now the form of the constants in the above system is shaped by the fact that we have set the problem for convenience in nondimensional units. For the reader interested in the connection to conventional units of measurement we describe briefly the relationship between the two systems of units. The problem was set in nondimensional form using the transformations

$$
t=\frac{k_{1} \hat{t}}{\rho_{1} c_{1} L^{2}}, \quad x=\frac{\hat{x}}{L},
$$

where $\hat{t}$ (sec) and $\hat{x}(\mathrm{~cm})$ are the time and space coordinates, $L(\mathrm{~cm})$ is the total length of the system, $c_{i}, i=1,2$, are the heat capacities (cal/deg-gr), $k_{i}, i=1,2$, are the heat conductivities (cal/deg-sec-cm) and $\rho_{i}, i=1,2$, are the densities $(\mathrm{gr} / \mathrm{cm})$ of the two rods. We suppose that the first rod extends over $0 \leq \hat{x} \leq L_{1}$, while the second rod extends over $L_{2} \leq \hat{x} \leq L$. Consequently, $l_{1}=L_{1} / L$ and $l_{2}=$ $\left(L-L_{2}\right) / L$. If we allow $\hat{\theta}_{i}\left({ }^{\circ} \mathrm{C}\right)$ to denote the temperature of $\operatorname{rod} i(i=1,2)$, then

$$
\theta_{i}=\frac{\hat{\theta}_{i}-\theta_{0}}{\theta_{*}-\theta_{0}},
$$


where $\theta_{0}$ is the uniform temperature of the reference configuration and $\theta_{*}$ can be taken to be any value of $\hat{\theta}_{1}(0, \cdot)$. If we allow $\hat{u}_{i}(\mathrm{~cm})$ to denote the displacement of $\operatorname{rod} i$, then

$$
u_{1}=\left(\frac{\left(\lambda_{1}+2 \mu_{1}\right) \theta_{0}}{\left(\theta_{*}-\theta_{0}\right)^{2} \rho_{1} c_{1} L^{2}}\right)^{1 / 2} \hat{u}_{1}, \quad u_{2}=\left(\frac{\theta_{0} k_{1}\left(\lambda_{2}+2 \mu_{2}\right)}{\left(\theta_{*}-\theta_{0}\right)^{2} \rho_{1} c_{1} k_{2} L^{2}}\right)^{1 / 2} \hat{u}_{2} .
$$

Here $\lambda_{i}$ and $\mu_{i}$ are the elastic moduli. The constants $a_{1}, a_{2}$, and $d$ that appear in the system are given by

$$
a_{1}^{2}=\frac{\theta_{0} \alpha_{1}^{2}\left(3 \lambda_{1}+2 \mu_{1}\right)^{2}}{\rho_{1} c_{1}\left(\lambda_{1}+2 \mu_{1}\right)}, \quad a_{2}^{2}=\frac{\theta_{0} \alpha_{2}^{2} k_{1}\left(3 \lambda_{2}+2 \mu_{2}\right)^{2}}{\rho_{1} c_{1} k_{2}\left(\lambda_{2}+2 \mu_{2}\right)},
$$

and

$$
d=\frac{\rho_{2} c_{2} k_{1}}{\rho_{1} c_{1} k_{2}}
$$

where $\alpha_{i}$ are the coefficients of thermal expansion for each rod. For most materials that arise in practice $a_{1}$ and $a_{2}$ are very small (see, e.g., [15]).

Returning to our description of the model, we now formulate our initial and boundary conditions. At the fixed ends $x=0$ and $x=1$ we suppose that the temperature is prescribed. Thus

$$
\begin{array}{cc}
\theta_{1}(0, t)=f_{0}(t), & \theta_{2}(1, t)=f_{1}(t), \quad 0 \leq t \leq T, \\
u_{1}(0, t)=0, & u_{2}(1, t)=0, \quad 0 \leq t \leq T .
\end{array}
$$

The initial temperatures of each rod are also prescribed:

$$
\theta_{1}(x, 0)=\varphi(x), \quad 0 \leq x \leq l_{1} ; \quad \theta_{2}(x, 0)=\varphi_{2}(x), \quad l_{2} \leq x \leq 1 .
$$

We remark that since the process is being treated as quasistatic we cannot prescribe the initial displacement. We turn next to describe the heat exchange condition at the free ends:

$$
\begin{aligned}
k_{1} \theta_{1 x}\left(l_{1}, t\right) & =k_{2} \theta_{2 x}\left(l_{2}, t\right), \quad 0<t \leq T, \\
-k_{1} \theta_{1 x}\left(l_{1}, t\right) & =k\left(\theta_{1}\left(l_{1}, t\right)-\theta_{2}\left(l_{2}, t\right)\right), \quad 0<t \leq T .
\end{aligned}
$$

Here $k_{1}$ and $k_{2}$ are the heat condition coefficients and $k$ is the heat exchange coefficient. We shall treat all three as positive constants, although we will remark on the case when $k$ is nonconstant at the end of this section. Equation (2.8) is a reasonable model for the heat exchange under the assumption that the heat flux leaving one rod enters the other rod without any losses and is proportional to the temperature difference of the free edges. To model the mechanical contact at the free edges we note first (see, e.g., [6,9]), that the stresses in the rods are given by

$$
\sigma_{i}(x, t)=\beta_{i}\left(u_{i x}(x, t)-a_{i} \theta_{i}(x, t)\right), \quad i=1,2 .
$$

Here $\beta_{1}$ and $\beta_{2}$ are positive constants, given by

$$
\beta_{1}=\left(\theta_{*}-\theta_{0}\right)\left[\frac{\left(\lambda_{1}+2 \mu_{1}\right) c_{1}}{\theta_{0}}\right]^{1 / 2}, \quad \beta_{2}=\left(\theta_{*}-\theta_{0}\right)\left[\frac{\left(\lambda_{2}+2 \mu_{2}\right) c_{1} k_{2}}{\theta_{0} k_{1}}\right]^{1 / 2} .
$$


It follows from (2.2), (2.4), and (2.9) that both stresses are independent of $x$. Consequently, the values of both functions are determined by what happens at the free ends. At those ends we assume that when there is no contact $\sigma_{1}=\sigma_{1}\left(l_{1}, \cdot\right)=0$ and $\sigma_{2}=\sigma_{2}\left(l_{2}, \cdot\right)=0$, and when there is contact $\sigma_{1}\left(l_{1}, \cdot\right)=\sigma_{2}\left(l_{2}, \cdot\right)$. In both cases then we have that $\sigma_{1}(t)=\sigma_{2}(t)=\sigma(t)$. Our condition for the mechanical contact at the free ends is of the Signorini type, namely

$$
\begin{gathered}
u_{1}\left(l_{1}, t\right) \leq u_{2}\left(l_{2}, t\right)+g, \quad 0 \leq t \leq T, \\
\sigma(t) \leq 0, \quad 0<t \leq T, \\
\sigma(t)\left[g+u_{2}\left(l_{2}, t\right)-u_{1}\left(l_{1}, t\right)\right]=0, \quad 0<t \leq T .
\end{gathered}
$$

(2.10) ensures that there is no penetration at the free ends, i.e., the right edge of the left rod is always to the left of the left edge of the right rod. (2.11) ensures that at all times the stress is compressive in both rods, and finally (2.12) says that when the rods are not in contact the stress is zero. This completes our description of the mathematical model.

The problem of thermoelastic contact of two dissimilar rods thus consists of (2.1)(2.8) and (2.10)-(2.12). A solution to the problem consists of $\left(\theta_{1}, u_{1}, \theta_{2}, u_{2}\right)$, where $\theta_{1}$ and $u_{1}$ are defined on $\Omega_{1 T}=\left(0, l_{1}\right) \times(0, T)$ and $\theta_{2}$ and $u_{2}$ are defined on $\Omega_{2 T}=\left(l_{2}, 1\right) \times(0, T)$. The expected regularity of a solution, i.e., the spaces in which we seek these functions, will be discussed in the next section.

As in the case of the one-rod problem (see, e.g., [15] or [2]) and the axially symmetric problem (see $[3,20,21]$ ), we are able to eliminate the displacements from the system (2.1)-(2.8) and (2.10)-(2.12) (even though the coupling constants are nonzero) and to reduce the problem to solving a parabolic system that contains only the temperatures. Once the thermal problem has been solved, the displacements may be found by quadrature. We now show informally how the elimination is effected. We begin by integrating $(2.2)$ with respect to $x$ over $[0, x]$ to obtain

$$
u_{1 x}(x, t)=a_{1} \theta_{1}(x, t)-a_{1} f_{0}(t)+u_{1 x}(0, t) .
$$

Another integration with respect to $x$ over $\left(0, l_{1}\right)$ results in

$$
u_{1}\left(l_{1}, t\right)=a_{1} \int_{0}^{l_{1}} \theta_{1}(x, t) d x-a_{1} l_{1} f_{0}(t)+l_{1} u_{1 x}(0, t) .
$$

Eliminating $u_{1 x}(0, t)$ from both equations yields

$$
u_{1 x}(x, t)=a_{1} \theta_{1}(x, t)-\frac{a_{1}}{l_{1}} \int_{0}^{l_{1}} \theta_{1}(\xi, t) d \xi+\frac{1}{l_{1}} u_{1}\left(l_{1}, t\right) .
$$

This, together with (2.9), implies that

$$
\left.\beta_{1}^{-1} \sigma_{1}(t)=u_{1 x}\left(l_{1}, t\right)-a_{1} \theta_{1}\left(l_{1}, t\right)\right)=\frac{1}{l_{1}} u_{1}\left(l_{1}, t\right)-\frac{a_{1}}{l_{1}} \int_{0}^{l_{1}} \theta_{1}(\xi, t) d \xi .
$$

Consequently, conditions (2.11) and (2.12) may be written equivalently as

$$
u_{1}\left(l_{1}, t\right) \leq a_{1} \int_{0}^{l_{1}} \theta_{1}(\xi, t) d \xi
$$


and

$$
\left[u_{1}\left(l_{1}, t\right)-u_{2}\left(l_{2}, t\right)-g\right]\left[u_{1}\left(l_{1}, t\right)-a_{1} \int_{0}^{l_{1}} \theta_{1}(\xi, t) d \xi\right]=0 .
$$

Now (2.10), (2.15), and this last equation together imply that

$$
u_{1}\left(l_{1}, t\right)=\min \left\{a_{1} \int_{0}^{l_{1}} \theta_{1}(\xi, t) d \xi, u_{2}\left(l_{2}, t\right)+g\right\} .
$$

Similarly, we find that

$$
\begin{aligned}
& \beta_{2}^{-1} \sigma_{2}(t)=u_{2 x}\left(l_{2}, t\right)-a_{2} \theta_{2}\left(l_{2}, t\right) \\
& -\frac{1}{1-l_{2}}\left(u_{2}\left(l_{2}, t\right)+a_{2} \int_{l_{2}}^{1} \theta_{2}(\xi, t) d \xi\right) .
\end{aligned}
$$

To simplify the notation below we introduce

$$
\boldsymbol{\Theta}_{1}(t)=a_{1} \int_{0}^{l_{1}} \theta_{1}(\xi, t) d \xi \quad \text { and } \quad \Theta_{2}(t)=a_{2} \int_{l_{2}}^{1} \theta_{2}(\xi, t) d \xi
$$

The fact that $\sigma_{1}(t)=\sigma_{2}(t)$ then implies that

$$
u_{1}\left(l_{1}, t\right)-\boldsymbol{\Theta}_{1}(t)=-\lambda\left(u_{2}\left(l_{2}, t\right)+\Theta_{2}(t)\right),
$$

where we have set $\lambda=\beta_{2} l_{1} /\left(\beta_{1}\left(1-l_{2}\right)\right)$. Since $\sigma_{2}(t) \leq 0$, it follows that

$$
u_{2}\left(l_{2}, t\right) \geq-\Theta_{2}(t) \text {. }
$$

and this, together with (2.10) and (2.12), implies that

$$
u_{2}\left(l_{2}, t\right)=\max \left\{u_{1}\left(l_{1}, t\right)-g,-\Theta_{2}(t)\right\} .
$$

Differentiating (2.13) and using (2.16), we find that

$$
-a_{1} u_{1 x t}(x, t)=-a_{1}^{2} \theta_{1 t}=\frac{a_{1}}{l_{1}} \frac{d}{d t} \min \left\{u_{2}\left(l_{2}, t\right)+g-\Theta_{1}(t), 0\right\} .
$$

Next, we consider the right-hand side of this expression when $u_{2}\left(l_{2}, t\right)+g-\Theta_{1}(t) \leq$ 0 . In this case it follows from (2.16) that $u_{1}\left(l_{1}, t\right)=u_{2}\left(l_{2}, t\right)+g$, and then (2.18) implies that

$$
u_{2}\left(l_{2}, t\right)+g-\Theta_{1}(t)=\frac{\lambda}{1+\lambda}\left[g-\Theta_{1}(t)-\Theta_{2}(t)\right] .
$$

On the other hand, if $u_{2}\left(l_{2}, t\right)+g-\Theta_{1}(t)>0$, then it follows from (2.16) and (2.18) that $u_{1}\left(l_{1}, t\right)=\Theta_{1}(t)$ and $u_{2}\left(l_{2}, t\right)=-\Theta_{2}(t)$. We can thus conclude that

$$
\begin{aligned}
\min & \left\{u_{2}\left(l_{2}, t\right)+g-\boldsymbol{\Theta}_{1}(t), 0\right\} \\
& =\frac{\lambda}{1+\lambda} \min \left\{g-\boldsymbol{\Theta}_{1}(t)-\boldsymbol{\Theta}_{2}(t), 0\right\} .
\end{aligned}
$$

In the same way (2.17) implies that

$$
-a_{2} u_{2 x t}(x, t)=\frac{a_{2}}{1-l_{2}} \frac{d}{d t}\left(u_{2}\left(l_{2}, t\right)+\Theta_{2}(t)\right)-a_{2}^{2} \theta_{2 t}(x, t) .
$$


Now (2.19) yields

$$
\begin{aligned}
u_{2}\left(l_{2}, t\right)+\Theta_{2}(t) & =\max \left\{u_{1}\left(l_{1}, t\right)-g,-\Theta_{2}(t)\right\}+\Theta_{2}(t) \\
& =\max \left\{u_{1}\left(l_{1}, t\right)-g+\Theta_{2}(t), 0\right\} .
\end{aligned}
$$

Again, if we consider the case when $u_{1}\left(l_{1}, t\right)-g+\Theta_{2}(t) \geq 0$, then (2.19) implies that $u_{2}\left(l_{2}, t\right)=u_{1}\left(l_{1}, t\right)-g$, and so $(2.18)$ yields

$$
u_{1}\left(l_{1}, t\right)-g+\boldsymbol{\Theta}_{2}(t)=-\frac{1}{1+\lambda}\left(g-\boldsymbol{\Theta}_{1}(t)-\boldsymbol{\Theta}_{2}(t)\right) .
$$

Consequently, we can conclude that

$$
\begin{aligned}
\max & \left\{u_{1}\left(l_{1}, t\right)-g+\Theta_{1}(t), 0\right\} \\
& =-\frac{1}{1+\lambda} \min \left\{g-\Theta_{1}(t)-\Theta_{2}(t), 0\right\} .
\end{aligned}
$$

Using the results of $(2.20)-(2.23)$ in (2.1) and (2.3) leads to

$$
\begin{aligned}
(1+ & \left.a_{1}^{2}\right) \theta_{1 t}-\theta_{1 x x} \\
& =-\frac{a_{1} \lambda}{l_{2}(1+\lambda)} \frac{d}{d t} \min \left\{g-\Theta_{1}(t)-\Theta_{2}(t), 0\right\}
\end{aligned}
$$

and

$$
\begin{aligned}
(d+ & \left.a_{2}^{2}\right) \theta_{2 t}-\theta_{2 x x} \\
& =-\frac{a_{2} \lambda}{(1+\lambda)\left(1-l_{2}\right)} \frac{d}{d t} \min \left\{g-\Theta_{1}(t)-\Theta_{2}(t), 0\right\} .
\end{aligned}
$$

The thermal problem thus consists of (2.24), (2.25), (2.5), (2.7), and (2.8). Once it has been solved the displacements of the edges may be found from

$$
u_{1}\left(l_{1}, t\right)=\min \left\{\frac{1}{1+\lambda}\left(\lambda g-\lambda \Theta_{2}(t)+\Theta_{1}(t)\right), \Theta_{1}(t)\right\}
$$

and

$$
u_{2}\left(l_{2}, t\right)=\max \left\{\frac{-1}{1+\lambda}\left(g+\lambda \Theta_{2}(t)-\Theta_{1}(t)\right),-\Theta_{2}(t)\right\} .
$$

The steady state of the thermal problem is easily obtained by setting all the time derivatives equal to zero. Then we find that

$$
\bar{\theta}_{1}(x)=1-A x, \quad \bar{\theta}_{2}(x)=K A(1-x),
$$

where $K=k_{1} / k_{2}$ and $A=k /\left(k_{1}+k\left(l_{1}+K\left(1-l_{2}\right)\right)\right)$. To find the corresponding displacements we first compute

$$
\begin{aligned}
& \Theta_{1}=a_{1} \int_{0}^{l_{1}} \bar{\theta}_{1}(x) d x=a_{1} l_{1}-\frac{1}{2} a_{1} A l_{1}^{2}, \\
& \boldsymbol{\Theta}_{2}=a_{2} \int_{l_{2}}^{1} \bar{\theta}_{2}(x) d x=\frac{1}{2} a_{2} K A\left(1-l_{2}\right)^{2},
\end{aligned}
$$


and then observe that contact occurs exactly when $G \leq 0$, where

$$
G=g-\Theta_{1}-\Theta_{2}=g-a_{1} l_{1}+\frac{1}{2} a_{1} A l_{1}^{2}-\frac{1}{2} a_{2} K A\left(1-l_{2}\right)^{2} .
$$

Consequently,

$$
\begin{aligned}
& u_{1}(x)=\left(a_{1}+\sigma\right) x-\frac{1}{2} a_{1} A x^{2}, \\
& u_{2}(x)=-\sigma(1-x)-\frac{1}{2} a_{2} K A(1-x)^{2},
\end{aligned}
$$

where $\sigma$, the contact pressure, is given by

$$
\sigma=\frac{\beta_{1} \beta_{2}}{l_{1} \beta_{2}+\beta_{1}\left(1-l_{2}\right)} \min \{G, 0\} .
$$

Finally, we remark that, although in this paper we have taken the heat coefficient $k$ to be constant, more complicated forms for $k$ have been considered in the one-rod case [1, 2] and the axially-symmetric case [3]. In particular, these papers have treated the case when $k$ is a continuously differentiable function that is allowed to depend on the gap size when there is no contact and on the contact pressure when there is contact. In the context of our current problem this would amount to letting $k$ be a continuously differentiable function of $g-\Theta_{1}(t)-\Theta_{2}(t)$. We leave this possibility for another study.

3. The main results. In this section we give a precise statement of the parabolic problem for the temperatures, and of our existence and uniqueness results. First we describe the function spaces in which we expect to find our solutions. Recall that $\Omega_{1 T}=\left(0, l_{1}\right) \times(0, T)$ and $\Omega_{2 T}=\left(l_{2}, 1\right) \times(0, T)$ for $T>0$. From the form of equations (2.23) and (2.24) it is natural to seek solutions $\theta_{1}$ and $\theta_{2}$ that lie respectively in the Sobolev spaces $W_{2}^{2,1}\left(\Omega_{1 T}\right)$ and $W_{2}^{2,1}\left(\Omega_{2 T}\right)$. These spaces consist of all square-integrable functions that possess generalized square-integrable secondorder spatial and first-order time derivatives. The norms in these Hilbert spaces are given by

$$
\|\theta\|_{W^{2,1}\left(\Omega_{i T}\right)}^{2}=\int_{\Omega_{i T}}\left(\theta_{t}^{2}+\theta_{x}^{2}+\theta_{x x}^{2}+\theta^{2}\right) d x d t, \quad i=1,2 .
$$

If $\theta_{i} \in W_{2}^{2,1}\left(\Omega_{i T}\right)$, then it is known [13, p. 9] that the boundary functions $\theta_{1}(0, \cdot)$ and $\theta_{2}(1, \cdot)$ lie in $H^{3 / 4}(0, T)$ while $\theta_{1 x}(0, \cdot)$ and $\theta_{2 x}(1, \cdot)$ lie in $H^{1 / 4}(0, T)$. The traces for fixed $t$ belong to $H^{1}$, i.e., $\theta_{1}(\cdot, t) \in H^{1}\left(0, l_{1}\right)$ and $\theta_{2}(\cdot, t) \in H^{1}\left(l_{2}, 1\right)$. These facts imply that the initial-boundary data must have the degree of regularity described below. For further properties of Sobolev spaces as well as any unexplained notation we refer the reader to the standard references $[12,13]$.

We can now give a complete description of the problem that we will consider in this paper:

Given $\varphi_{1} \in H^{1}\left(0, l_{1}\right)$ and $\varphi_{2} \in H^{1}\left(l_{2}, 1\right)$ such that $\varphi_{1}(0)=0$ and $\varphi_{2}(1)=0$, find a pair $\left(\theta_{1}, \theta_{2}\right)=W_{2}^{2,1}\left(\Omega_{1 T}\right) \times W_{2}^{2,1}\left(\Omega_{2 T}\right)$ such that

$$
\begin{aligned}
& \left(1+a_{1}^{2}\right) \theta_{1 t}-\theta_{1 x x} \\
& \quad=-\frac{a_{1} \lambda}{l_{1}(1+\lambda)} \frac{d}{d t} \min \left\{g-\Theta_{1}(t)-\Theta_{2}(t), 0\right\}, \quad \text { in } \Omega_{1 T},
\end{aligned}
$$




$$
\begin{gathered}
\left(d+a_{2}^{2}\right) \theta_{2 t}-\theta_{2 x x} \\
=-\frac{a_{2} \lambda}{\left(1-l_{2}\right)(1+\lambda)} \frac{d}{d t} \min \left\{g-\theta_{1}(t)-\theta_{2}(t), 0\right\}, \quad \text { in } \Omega_{2 T}, \\
\theta_{1}(x, 0)=\varphi_{1}(x), \quad 0 \leq x \leq l_{1} ; \quad \theta_{2}(x, 0)=\varphi_{2}(x), \quad l_{2} \leq x \leq 1, \\
\theta_{1}(0, t)=0, \quad 0 \leq t \leq T ; \quad \theta_{2}(1, t)=0, \quad 0 \leq t \leq T, \\
k_{1} \theta_{1 x}\left(l_{1}, t\right)=k_{2} \theta_{2 x}\left(l_{2}, t\right), \quad 0<t \leq T, \\
-k_{1} \theta_{1 x}\left(l_{1}, t\right)=k\left(\theta_{1}\left(l_{1}, t\right)-\theta_{2}\left(l_{2}, t\right)\right), \quad 0<t \leq T .
\end{gathered}
$$

Here the derivatives are understood in the distributional sense and the conditions (3.3)-(3.6) hold in the trace sense. Recall also that

$$
\Theta_{1}(t)=a_{1} \int_{0}^{l_{1}} \theta_{1}(\xi, t) d \xi, \quad \Theta_{2}(t)=a_{2} \int_{l_{2}}^{1} \theta_{2}(\xi, t) d \xi
$$

Note that in (3.4) we have assumed, for the sake of simplicity, that $\theta_{1}(0, \cdot)$ and $\theta_{2}(1, \cdot)$ are both zero. We can, in fact, take them to be arbitrary functions in $H^{1}(0, T)$, but have refrained from doing so in order to avoid cluttering up our proofs with unnecessary details. The main problem in dealing with the above system is that the right-hand sides of (3.1) and (3.2) are nonlinear and nonlocal functions of $\theta_{1}$ and $\theta_{2}$. Moreover, as was first pointed out in [16], the operator $\left(\theta_{1}, \theta_{2}\right) \rightarrow \frac{d}{d t} \min \left\{g-\Theta_{1}(t)-\Theta_{2}(t), 0\right\}$ is not even Lipschitz continuous as an operator from $H^{1}\left(\Omega_{1 T}\right) \times H^{1}\left(\Omega_{2 T}\right)$ to $L^{2}(0, T)$. Consequently, we cannot simply quote standard results in the parabolic theory to obtain a solution. Instead, we derive a priori estimates peculiar to this particular problem and use a fixed-point argument and the functional method of Ladyzhenskaya [11]. Our main result is the following.

Theorem 3.1. Given $\varphi_{1} \in H^{1}\left(0, l_{1}\right)$ and $\varphi_{2} \in H^{1}\left(l_{2}, 1\right)$ such that $\varphi_{1}(0)=0$ and $\varphi_{2}(1)=0$, there exists a unique solution $\left(\theta_{1}, \theta_{2}\right) \in W_{2}^{2,1}\left(\Omega_{1 T}\right) \times W_{2}^{2,1}\left(\Omega_{2 T}\right)$ to problem (3.1)-(3.7), provided that $a_{1}$ and $a_{2}$ are sufficiently small.

In fact, our proof of this theorem requires $a_{1}$ and $a_{2}$ to satisfy the condition:

$$
\begin{aligned}
\frac{a_{1}^{4}}{l_{1}^{2}\left(b_{1}+a_{1}^{2}\right)^{2}} & +\frac{a_{1}^{2} a_{2}^{2} k_{2}}{k_{1} l_{1}^{2}\left(b_{1}+a_{1}^{2}\right)\left(b_{2}+a_{2}^{2}\right)}+\frac{a_{1}^{2} a_{2}^{2} k_{1}}{k_{2}\left(1-l_{2}\right)^{2}\left(b_{1}+a_{1}^{2}\right)\left(b_{2}+a_{2}^{2}\right)} \\
& +\frac{a_{2}^{4}}{\left(1-l_{2}\right)^{2}\left(b_{2}+a_{2}^{2}\right)^{2}} \leq \frac{1}{2}\left(1+\lambda^{-1}\right)^{2} .
\end{aligned}
$$

Since for most materials that occur in practice $a_{1}$ and $a_{2}$ are small, it is possible to satisfy the hypotheses of this theorem. The proof of this theorem will be given in the next section.

To conclude this section we investigate the relationship between the solution to (3.1)-(3.7) given by the above theorem and a sufficiently regular solution to our original problem (2.1)-(2.12). For this purpose we introduce the nonstandard spaces

$$
W_{i}=\left\{u_{i} \in L^{2}\left(\Omega_{i T}\right): u_{i x}, u_{i x t}, u_{i x x} \in L^{2}\left(\Omega_{i T}\right)\right\}
$$


equipped with the norms

$$
\left\|u_{i}\right\|_{W_{i}}^{2}=\int_{\Omega_{i T}}\left(u_{i}^{2}+u_{i x}^{2}+u_{i t x}^{2}+u_{i x x}^{2}\right) d r d t, \quad i=1,2 .
$$

It is now straightforward to establish the following equivalence between solutions in a way similar to that done in [15] for the case of a single rod.

THEOREM 3.2. If $\left\{\theta_{1}, u_{1}, \theta_{2}, u_{2}\right\} \in W_{2}^{2,1}\left(\Omega_{1 T}\right) \times W_{1} \times W_{2}^{2,1}\left(\Omega_{2 T}\right) \times W_{2}$ satisfies (2.1)-(2.12), then $\left\{\theta_{1}, \theta_{2}\right\}$ satisfies (3.1)-(3.7). Conversely, if $\left\{\theta_{1}, \theta_{2}\right\} \in W_{2}^{2,1}\left(\Omega_{1 T}\right)$ $\times W_{2}^{2,1}\left(\Omega_{2 T}\right)$ is the solution to (3.1)-(3.7) and if $u_{1}$ and $u_{2}$ are given by

$$
\begin{aligned}
u_{1}(x, t)= & a_{1} \int_{0}^{x} \theta_{1}(\xi, t) d \xi \\
& +\frac{\lambda x}{(1+\lambda) l_{1}} \min \left\{g-\Theta_{1}(t)-\Theta_{2}(t), 0\right\}
\end{aligned}
$$

and

$$
\begin{aligned}
u_{2}(x, t)= & -a_{2} \int_{x}^{1} \theta_{2}(\xi, t) d \xi \\
& -\frac{1-x}{\left(1-l_{2}\right)(1+\lambda)} \min \left\{g-\Theta_{1}(t)-\Theta_{2}(t), 0\right\}
\end{aligned}
$$

then $\left\{\theta_{1}, u_{1}, \theta_{2}, u_{2}\right\}$ lies in $W_{2}^{2,1}\left(\Omega_{1 T}\right) \times W_{1} \times W_{2}^{2,1}\left(\Omega_{2 T}\right) \times W_{2}$ and satisfies (2.1)(2.12).

In fact we can make a stronger statement about the regularity of $u_{1}$ and $u_{2}$.

Corollary 3.3. If $\left\{\theta_{1}, \theta_{1}\right\}$ is a solution of $(3.1)-(3.7)$ in $W_{2}^{2,1}\left(\Omega_{1 T}\right) \times W_{2}^{2,1}\left(\Omega_{2 T}\right)$ then $u_{1} \in W_{2}^{3,1}\left(\Omega_{1 T}\right)$ and $u_{2} \in W_{2}^{3,1}\left(\Omega_{2 T}\right)$.

Proof. Corollary 3.3 follows directly from (3.8), (3.9), and the regularity of $\theta_{1}$ and $\theta_{2}$.

4. Proofs of existence and uniqueness. In this section we prove Theorem 3.1. Existence of solutions will be established by using the Schauder fixed-point theorem and the solution to an auxiliary problem. The solution to the auxiliary problem in turn is based on the functional method of Ladyzhenskaya [11]. Uniqueness of solutions will then follow from the a priori estimates obtained during the course of the existence proof.

We first introduce some notation to cast our equations in a form that makes for greater typographical efficiency. Let $I_{1}=\left(0, l_{1}\right), I_{2}=\left(l_{2}, 1\right), b_{1}=1$, and $b_{2}=d$. Taking $\varphi_{i} \in H^{1}\left(I_{i}\right), i=1,2$, with $\varphi_{1}(0)=\varphi_{2}(1)=0$, and fixing positive constants $k, k_{1}$, and $k_{2}$, we seek $\left(\theta_{1}, \theta_{2}\right)$ in $W_{2}^{2,1}\left(\Omega_{1 T}\right) \times W_{2}^{2,1}\left(\Omega_{2 T}\right)$ that satisfies the following conditions:

$$
\begin{aligned}
\left(b_{i}+a_{i}^{2}\right) \theta_{i t}-\theta_{i x x} & =\alpha_{i} h \quad \text { in } \Omega_{i T}, i=1,2 \\
\theta_{i}(x, 0) & =\varphi_{i}(x), \quad x \in I_{i}, \quad i=1,2, \\
\theta_{1}(0, t) & =\theta_{2}(1, t)=0, \quad t \in(0, T), \\
-k_{i} \theta_{i x}\left(l_{i}, t\right) & =k\left(\theta_{1}\left(l_{1}, t\right)-\theta_{2}\left(l_{2}, t\right)\right), \quad t \in(0, T), \quad i=1,2 ;
\end{aligned}
$$


here and below $\alpha_{1}=a_{1} \lambda l_{1}^{-1}(1+\lambda)^{-1}, \alpha_{2}=a_{2} \lambda(1+\lambda)^{-1}\left(1-l_{2}\right)^{-1}$, and

$$
h=\frac{d}{d t} \max \left\{a_{1} \int_{0}^{l_{1}} \theta_{1}(\xi, \cdot) d \xi+a_{2} \int_{l_{2}}^{1} \theta_{2}(\xi, \cdot) d \xi-g, 0\right\} .
$$

The following lemma gives the solution to an auxiliary problem.

LEMMA 4.1. Let $f_{i} \in L^{2}\left(\Omega_{i T}\right), i=1,2$. Then there exists a unique pair $\left(\theta_{1}, \theta_{2}\right)$ in $W_{2}^{2,1}\left(\Omega_{1 T}\right) \times W_{2}^{2,1}\left(\Omega_{2 T}\right)$ such that

$$
\left(b_{i}+a_{i}^{2}\right) \theta_{i t}-\theta_{i x x}=f_{i} \quad \text { in } \Omega_{i T}, i=1,2,
$$

and

$$
\left(\theta_{1}, \theta_{2}\right) \text { satisfies }(4.2)-(4.4) \text {. }
$$

Proof. Everywhere below $i=1,2$; so we will dispense with writing this explicitly. We introduce the following spaces and norms. Let

$$
\begin{aligned}
H & =\left\{\left(u_{1}, u_{2}\right) \in W_{2}^{2,1}\left(\Omega_{1 T}\right) \times W_{2}^{2,1}\left(\Omega_{2 T}\right):\left(u_{1}, u_{2}\right) \text { satisfies (4.3), (4.4) },\right. \\
H_{1} & =\left\{f \in H^{1}\left(0, l_{1}\right): f(0)=0\right\}, \\
H_{2} & =\left\{f \in H^{1}\left(l_{2}, 1\right): f(1)=0\right\},
\end{aligned}
$$

and

$$
X=L^{2}\left(\Omega_{1 T}\right) \times L^{2}\left(\Omega_{2 T}\right) \times H_{1} \times H_{2} .
$$

On $H_{1}$ and $H_{2}$, we define the norms

$$
\|f\|_{H_{i}}^{2}=2 k f\left(l_{i}\right)^{2}+k_{i}\left\|f^{\prime}\right\|_{L^{2}\left(I_{i}\right)}^{2} .
$$

On $H$, we define the norm

$$
\begin{aligned}
\left\|\left(u_{1}, u_{2}\right)\right\|_{H}^{2}=\frac{1}{1+T} \sum_{i} k_{i}\left(\left(b_{i}+a_{i}^{2}\right)\left\|u_{i t}\right\|_{L^{2}\left(\Omega_{i T}\right)}^{2}+\left\|u_{i x}\right\|_{L^{2}\left(\Omega_{i T}\right)}^{2}\right. & \left.+\frac{1}{\left(b_{i}+a_{i}^{2}\right)}\left\|u_{i x x}\right\|_{L^{2}\left(\Omega_{i T}\right)}^{2}\right) .
\end{aligned}
$$

On $X$, we define the norm

$$
\left\|\left(v_{1}, v_{2}, g_{1}, g_{2}\right)\right\|_{X}^{2}=\sum_{i}\left(\frac{k_{i}}{b_{i}+a_{i}^{2}}\left\|v_{i}\right\|_{L^{2}\left(\Omega_{i T}\right)}^{2}+\left\|g_{i}\right\|_{H_{i}}^{2}\right) .
$$

By the Poincare inequality, the norm on $H$ is equivalent to the restriction to $H$ of the standard product norm on $W_{2}^{2,1}\left(\Omega_{1 T}\right) \times W_{2}^{2,1}\left(\Omega_{2 T}\right)$. We note that, by continuity of the trace operators in $W_{2}^{2,1}\left(\Omega_{i T}\right), H$ is a Hilbert space in this norm.

Consider now the bounded linear operator $A: H \rightarrow X$ defined by

$$
A\left(u_{1}, u_{2}\right)=\left(\left(b_{1}+a_{1}^{2}\right) u_{1 t}-u_{1 x x},\left(b_{2}+a_{2}^{2}\right) u_{2 t}-u_{2 x x}, u_{1}(\cdot, 0), u_{2}(\cdot, 0)\right) \text {. }
$$

We will use the method described in Sec. III.2 of [11] to prove that $A$ is a bijection of $H$ onto $X$. This will establish Lemma 4.1. 
We prove first that $A$ is injective with closed range. We begin by supposing that $\left(u_{1}, u_{2}\right) \in H \cap\left(C^{\infty}\left(\bar{\Omega}_{1 T}\right) \times C^{\infty}\left(\bar{\Omega}_{2 T}\right)\right)$, and we set

$$
\left(b_{i}+a_{i}^{2}\right) u_{i t}-u_{i x x}=g_{i} .
$$

Squaring both sides of (4.7), multiplying through by $k_{i}\left(b_{i}+a_{i}^{2}\right)^{-1}$, and then integrating over $\Omega_{i t}=I_{i} \times(0, t)$ yields

$$
\begin{aligned}
& k_{i}\left(b_{i}+a_{i}^{2}\right)\left\|u_{i t}\right\|_{L^{2}\left(\Omega_{i t}\right)}^{2}+k_{i}\left\|u_{i x}(\cdot, t)\right\|_{L^{2}\left(I_{i}\right)}^{2}+\frac{k_{i}}{b_{i}+a_{i}^{2}}\left\|u_{i x x}\right\|_{L^{2}\left(\Omega_{i t}\right)}^{2} \\
& \quad+2 k_{i}(-1)^{i} \int_{0}^{t} u_{i x}\left(l_{i}, \tau\right) u_{i \tau}\left(l_{i}, \tau\right) d \tau \\
& =\frac{k_{i}}{b_{i}+a_{i}^{2}}\left\|g_{i}\right\|_{L^{2}\left(\Omega_{i t}\right)}^{2}+k_{i}\left\|u_{i x}(\cdot, 0)\right\|_{L^{2}\left(I_{i}\right)}^{2} .
\end{aligned}
$$

Using the boundary conditions on the elements of $H$, we see that the integral on the left-hand side of $(4.8)$ is

$$
2(-1)^{i+1} k \int_{0}^{t}\left(u_{1}\left(l_{1}, t\right)-u_{2}\left(l_{2}, t\right)\right) u_{i \tau}\left(l_{i}, \tau\right) d \tau
$$

Substituting (4.9) into (4.8) and then summing over $i$ produces

$$
\begin{aligned}
& k\left(u_{1}\left(l_{1}, t\right)-u_{2}\left(l_{2}, t\right)\right)^{2} \\
& \quad+\sum_{i}\left(k_{i}\left(b_{i}+a_{i}^{2}\right)\left\|u_{i t}\right\|_{L^{2}\left(\Omega_{i i}\right)}^{2}+k_{i}\left\|u_{i x}(\cdot, t)\right\|_{L^{2}\left(I_{i}\right)}^{2}+\frac{k_{i}}{b_{i}+a_{i}^{2}}\left\|u_{i x x}\right\|_{L^{2}\left(\Omega_{i t}\right)}^{2}\right) \\
& \quad \leq \sum_{i}\left(\frac{k_{i}}{b_{i}+a_{i}^{2}}\left\|g_{i}\right\|_{L^{2}\left(\Omega_{i t}\right)}^{2}+k_{i}\left\|u_{i x}(\cdot, 0)\right\|_{L^{2}\left(I_{i}\right)}^{2}+2 k u_{i}\left(l_{i}, 0\right)^{2}\right) .
\end{aligned}
$$

Consequently, for almost all $t$ in $(0, T)$,

$$
\begin{aligned}
& \sum_{i} k_{i}\left\|u_{i x}(\cdot, t)\right\|_{L^{2}\left(I_{i}\right)}^{2} \\
& \quad \leq \sum_{i}\left(\frac{k_{i}}{b_{i}+a_{i}^{2}}\left\|g_{i}\right\|_{L^{2}\left(\Omega_{i t}\right)}^{2}+k_{i}\left\|u_{i x}(\cdot, 0)\right\|_{L^{2}\left(I_{i}\right)}^{2}+2 k u_{i}\left(l_{i}, 0\right)^{2}\right) .
\end{aligned}
$$

Integrating this inequality over $(0, T)$, we obtain

$$
\begin{aligned}
& \sum_{i} k_{i}\left\|u_{i x}\right\|_{L^{2}\left(\Omega_{i i}\right)}^{2} \\
& \quad \leq T \sum_{i}\left(\frac{k_{i}}{b_{i}+a_{i}^{2}}\left\|g_{i}\right\|_{L^{2}\left(\Omega_{i i}\right)}^{2}+k_{i}\left\|u_{i x}(\cdot, 0)\right\|_{L^{2}\left(I_{i}\right)}^{2}+2 k u_{i}\left(l_{i}, 0\right)^{2}\right) .
\end{aligned}
$$

Setting $t=T$ in (4.10), adding the resulting inequality to (4.11), and using the definition of the norms on $H$ and $X$, we deduce that

$$
\left\|A\left(u_{1}, u_{2}\right)\right\|_{X} \geq\left\|\left(u_{1}, u_{2}\right)\right\|_{H}
$$


for all $\left(u_{1}, u_{2}\right) \in H \cap\left(C^{\infty}\left(\bar{\Omega}_{1 T}\right) \times C^{\infty}\left(\bar{\Omega}_{2 T}\right)\right.$. By density the above inequality holds for all $\left(u_{1}, u_{2}\right) \in H$. It follows that $A$ is injective with closed range.

Our next task is to establish that $A$ is surjective, which we will do by showing that the orthogonal complement in $X$ of the range of $A$ is null. Suppose then that $\left(w_{1}, w_{2}, \psi_{1}, \psi_{2}\right) \in X$ is orthogonal to the range of $A$. Then, for all $\left(v_{1}, v_{2}\right) \in H$,

$$
\begin{aligned}
0= & \sum_{i}\left(A_{i} \int_{\Omega_{i T}}\left(\left(b_{i}+a_{i}^{2}\right) v_{i t}-v_{i x x}\right) w_{i} d x d t\right. \\
& \left.+B_{i} v_{i}\left(l_{i}, 0\right) \psi_{i}\left(l_{i}\right)+D_{i} \int_{I_{i}} v_{i x}(\cdot, 0) \psi_{i}^{\prime} d x\right),
\end{aligned}
$$

where $A_{i}, B_{i}, D_{i}$ are positive constants depending only on the parameters of the system. We now choose $\tau \in(0, T)$ and construct functions $\bar{v}_{i} \in W_{2}^{2,0}\left(\Omega_{i T}\right)$ such that $\bar{v}_{i x x}=A_{i} k_{i}^{-1}\left(b_{i}+a_{i}^{2}\right) w_{i}$ on $\Omega_{i T}$ and $\left(\bar{v}_{1}, \bar{v}_{2}\right)$ satisfies (4.3), (4.4) on $(0, T)$. We then define

$$
v_{i}(x, t)= \begin{cases}\int_{\tau}^{t} \bar{v}_{i}(x, \sigma) d \sigma, & t \in[\tau, T], \\ 0, & t \in(0, \tau) .\end{cases}
$$

It follows that $\left(v_{1}, v_{2}\right) \in H$. Hence, using (4.12) and the fact that $v_{i}(\cdot, t)=0$ for $t \in(0, \tau)$, we obtain

$$
0=\sum_{i}\left(k_{i} \int_{\tau}^{T} \int_{I_{i}} v_{i t} v_{i x x t} d x d t-\frac{k_{i}}{b_{i}+a_{i}^{2}} \int_{\tau}^{T} \int_{I_{i}} v_{i x x} v_{i x x t} d x d t\right)
$$

By construction, the trace functions $v_{i x x}(\cdot, T)$ and $v_{i t}\left(l_{i}, \cdot\right)$ are well-defined elements of $L^{2}\left(I_{i}\right)$ and $L^{2}(0, T)$, respectively; so we can now use the boundary conditions for $\left(v_{1}, v_{2}\right)$ and the fact that $v_{i}(\cdot, \tau)=0$ to calculate that

$$
\int_{\tau}^{T} \int_{I_{i}} v_{i x x} v_{i x x t} d x d t=\frac{1}{2} \int_{I_{i}} v_{i x x}^{2}(x, T) d x
$$

$$
\begin{aligned}
& k_{i} \int_{\tau}^{T} \int_{I_{i}} v_{i t} v_{i x x t} d x d t \\
& \quad=(-1)^{i} k \int_{\tau}^{T} v_{i t}\left(l_{i}, t\right)\left(v_{1 t}\left(l_{1}, t\right)-v_{2 t}\left(l_{2}, t\right)\right) d t-k_{i} \int_{\tau}^{T} \int_{I_{i}} v_{i x t}^{2} d x d t .
\end{aligned}
$$

Substitution of (4.14) and (4.15) into (4.13) produces

$$
\begin{aligned}
0= & k \int_{\tau}^{T}\left(v_{1 t}\left(l_{1}, t\right)-v_{2 t}\left(l_{2}, t\right)\right)^{2} d t \\
& +\sum_{i}\left(k_{i} \int_{\tau}^{T} \int_{I_{i}} v_{i x t}^{2} d x d t+\frac{k_{i}}{2\left(b_{i}+a_{i}^{2}\right)} \int_{I_{i}} v_{i x x}^{2}(x, T) d x\right) .
\end{aligned}
$$

This implies that $v_{i x t}=0$ on $I_{i} \times(\tau, T)$, whence $w_{i}=v_{i x x t}=0$ on $I_{i} \times(\tau, T)$, and since $\tau$ is arbitrary, $w_{i}=0$ on $\Omega_{i T}$. Hence from (4.12), for all $\left(v_{1}, v_{2}\right) \in H$,

$$
0=\sum_{i}\left(B_{i} v_{i}\left(l_{i}, 0\right) \psi_{i}\left(l_{i}\right)+D_{i} \int_{I_{i}} v_{i x}(\cdot, 0) \psi_{i}^{\prime} d x\right) \text {. }
$$


We now claim that as $\left(v_{1}, v_{2}\right)$ ranges over $H,\left(v_{1}(\cdot, 0), v_{2}(\cdot, 0)\right)$ ranges over a subset of $\mathrm{H}_{1} \times \mathrm{H}_{2}$ that is dense in the product norm. To see this, note that it suffices to prove that if $\left(\psi_{1}, \psi_{2}\right)$ is any fixed element of $\left(C^{1}\left(\bar{I}_{1}\right) \cap H_{1}\right) \times\left(C^{1}\left(\bar{I}_{2}\right) \cap H_{2}\right)$, then there exists a sequence $\left(\varphi_{1 n}, \varphi_{2 n}\right)$ in this same space such that $-k_{i} \varphi_{i n}^{\prime}\left(l_{i}\right)=$ $k\left(\varphi_{1 n}\left(l_{1}\right)-\varphi_{2 n}\left(l_{2}\right)\right)$ for all $n$, and $\varphi_{i n} \rightarrow \psi_{i}$ strongly in $H_{i}$.

Let $p_{i}=\psi_{i}\left(l_{i}\right)$. It is straightforward to obtain sequences $\left(\varphi_{i n}\right) \subseteq C^{1}\left(\bar{I}_{i}\right)$ such that, for all $n, \varphi_{1 n}(0)=0, \varphi_{2 n}(1)=0, \varphi_{i n}\left(l_{i}\right)=p_{i}, \varphi_{i n}^{\prime}\left(l_{i}\right)=-k_{i}^{-1} k\left(p_{1}-p_{2}\right)$, and $\varphi_{i n} \rightarrow \psi_{i}$ strongly in $H_{i}$. The sequence $\left(\varphi_{1 n}, \varphi_{2 n}\right)$ thus meets all requirements. It now follows from (4.16) that $\psi_{i}=0$ in $H_{i}$. The orthogonal complement of the range of $A$ is therefore null, which proves that $A$ is surjective. This completes the proof of the lemma.

We can now prove the existence of solutions to problem (4.1)-(4.4). We begin by defining the operator $S: H \rightarrow H$ by $S\left(u_{1}, u_{2}\right)=\left(\theta_{1}, \theta_{2}\right)$ where $\left(\theta_{1}, \theta_{2}\right)$ is the unique solution to problem (4.5)-(4.6) with

$$
f_{i}=\alpha_{i} \frac{d}{d t} \max \left\{a_{i} \int_{0}^{l_{1}} u_{1}(\xi, \cdot) d \xi+a_{2} \int_{0}^{l_{2}} u_{2}(\xi, \cdot) d \xi-g, 0\right\} .
$$

For each $M>0$, define

$$
K_{M}=\left\{\left(u_{1}, u_{2}\right) \in H: u_{i}(\cdot, 0)=\varphi_{i}, \rho\left(u_{1}, u_{2}\right) \leq M\right\},
$$

where

$$
\begin{aligned}
\rho\left(u_{1}, u_{2}\right)^{2}=\sup _{0<t<T} \sum_{i} & \left(k_{i}\left(b_{i}+a_{i}^{2}\right)\left\|u_{i t}\right\|_{L^{2}\left(\Omega_{i t}\right)}^{2}\right. \\
& \left.+k_{i}\left\|u_{i x}(\cdot, t)\right\|_{L^{2}\left(I_{i}\right)}^{2}+\frac{k_{i}}{b_{i}+a_{i}^{2}}\left\|u_{i x x}\right\|_{L^{2}\left(\Omega_{i t}\right)}^{2}\right) .
\end{aligned}
$$

For $M$ sufficiently large the set $K_{M}$ is clearly a nonempty, bounded, closed, weakly compact, convex subset of $H$. We now wish to show that, for $M$ sufficiently large, the operator $S$ leaves $K_{M}$ invariant. This requires some estimates of $\left\|f_{i}\right\|_{L^{2}\left(\Omega_{i t}\right)}$. Thus,

$$
\begin{aligned}
\left\|f_{i}\right\|_{L^{2}\left(\Omega_{i t}\right)}^{2} \leq & 2 \alpha_{i}^{2}\left(a_{1}^{2} l_{1} \int_{0}^{T}\left|\int_{0}^{l_{1}} u_{1 t}(x, t) d x\right|^{2} d t\right. \\
& \left.+a_{2}^{2}\left(1-l_{2}\right) \int_{0}^{T}\left|\int_{l_{2}}^{1} u_{2 t}(x, t) d x\right|^{2} d t\right) \\
\leq & 2 \alpha_{i}^{2}\left(a_{1}^{2} l_{1}^{2}\left\|u_{1 t}\right\|_{L^{2}\left(\Omega_{1 T}\right)}^{2}+a_{2}^{2}\left(1-l_{2}\right)^{2}\left\|u_{2 t}\right\|_{L^{2}\left(\Omega_{2 T}\right)}^{2}\right) \\
\leq & 2 \alpha_{i}^{2}\left(a_{1}^{2}\left\|u_{1 t}\right\|_{L^{2}\left(\Omega_{1 T}\right)}^{2}+a_{2}^{2}\left\|u_{2 t}\right\|_{L^{2}\left(\Omega_{2 T}\right)}^{2}\right)
\end{aligned}
$$


Now let $\left(u_{1}, u_{2}\right) \in K_{M}$. Then, by (4.10) and (4.17),

$$
\begin{aligned}
\rho\left(S\left(u_{1}, u_{2}\right)\right)^{2} \leq & \left\|\varphi_{1}\right\|_{H_{1}}^{2}+\left\|\varphi_{2}\right\|_{H_{2}}^{2} \\
& +2\left(\frac{a_{1}^{2} \alpha_{1}^{2}}{\left(b_{1}+a_{1}^{2}\right)^{2}}+\frac{\alpha_{1}^{2} a_{2}^{2} k_{2}}{k_{1}\left(b_{1}+a_{1}^{2}\right)\left(b_{2}+a_{2}^{2}\right)}\right. \\
& \left.\quad+\frac{a_{1}^{2} \alpha_{2}^{2} k_{1}}{k_{2}\left(b_{1}+a_{1}^{2}\right)\left(b_{2}+a_{2}^{2}\right)}+\frac{a_{2}^{2}}{\left(b_{2}+a_{2}^{2}\right)}\right) M^{2} \\
\leq & C+\varepsilon\left(a_{1}, a_{2}, \alpha_{1}, \alpha_{2}\right) M^{2} .
\end{aligned}
$$

Thus, if we choose $a_{1}$ and $a_{2}$ so small that $\varepsilon\left(a_{1}, a_{2}, \alpha_{1}, \alpha_{2}\right)<1$, and then choose $M$ so that $M^{2} \geq C\left(1-\varepsilon\left(a_{1}, a_{2}, \alpha_{1}, \alpha_{2}\right)\right)^{-1}$, then the operator $S$ maps $K_{M}$ into $K_{M}$.

Now it can be shown in the same way as was done for the operators $F$ and $L$ found in [2, Sec. 4] that $S$ is weakly continuous on $K_{M}$. It follows that $S$ has a fixed point in $K_{M}$,which solves problem (4.1)-(4.4).

To establish uniqueness of solutions, we suppose that $\left(w_{1}, z_{1}\right)$ and $\left(w_{2}, z_{2}\right)$ are two strong solutions to (4.1)-(4.4), and set $w=w_{1}-w_{2}, z=z_{1}-z_{2}$, and

$$
u(x, t)=\int_{0}^{t} w(x, \tau) d \tau, \quad v(x, t)=\int_{0}^{t} z(x, \tau) d \tau .
$$

Then straightforward calculations show that $(u, v)$ satisfies $(4.1)-(4.4)$ with $\varphi_{1}=0$, $\varphi_{2}=0$, and with the right-hand side of (4.1) replaced by $\alpha_{i} F$, where

$$
\begin{aligned}
F= & \max \left\{a_{1} \int_{0}^{l_{1}} w_{1}(x, \cdot) d x+a_{2} \int_{l_{2}}^{1} z_{1}(x, \cdot) d x-g, 0\right\} \\
& -\max \left\{a_{1} \int_{0}^{l_{1}} w_{2}(x, \cdot) d x+a_{2} \int_{l_{2}}^{1} z_{2}(x, \cdot) d x-g, 0\right\} .
\end{aligned}
$$

Hence, by the same calculations that led to $(4.10)$, we obtain

$$
\begin{aligned}
& k_{1}\left(b_{1}+a_{1}^{2}\right)\left\|u_{t}\right\|_{L^{2}\left(\Omega_{1 T}\right)}^{2}+k_{2}\left(b_{2}+a_{2}^{2}\right)\left\|v_{t}\right\|_{L^{2}\left(\Omega_{2 T}\right)}^{2} \\
& \leq\left(\frac{k_{1} l_{1} \alpha_{1}^{2}}{b_{1}+a_{1}^{2}}+\frac{k_{2}\left(1-l_{2}\right) \alpha_{2}^{2}}{b_{2}+a_{2}^{2}}\right)\|F\|_{L^{2}(0, T)}^{2} .
\end{aligned}
$$

Since

it follows that

$$
\|F\|_{L^{2}(0, T)}^{2} \leq 2 a_{1}^{2} l_{1}\left\|u_{t}\right\|_{L^{2}\left(\Omega_{1 T}\right)}^{2}+2 a_{2}^{2}\left(1-l_{2}\right)\left\|v_{t}\right\|_{L^{2}\left(\Omega_{2 T}\right)}^{2},
$$

$$
\eta_{1}\left(a_{1}, a_{2}\right)\left\|u_{t}\right\|_{L^{2}\left(\Omega_{1 T}\right)}^{2}+\eta_{2}\left(a_{1}, a_{2}\right)\left\|v_{t}\right\|_{L^{2}\left(\Omega_{2 T}\right)}^{2} \leq 0
$$

where

$$
\eta_{1}\left(a_{1}, a_{2}\right)=k_{1}\left(b_{1}+a_{1}^{2}\right)-\frac{2 a_{1}^{2} \alpha_{1}^{2} k_{1}}{b_{1}+a_{1}^{2}}-\frac{2 a_{1}^{2} \alpha_{2}^{2} k_{2}}{b_{2}+a_{2}^{2}}
$$

and 


$$
\eta_{2}\left(a_{1}, a_{2}\right)=k_{2}\left(b_{2}+a_{2}^{2}\right)-\frac{2 a_{2}^{2} \alpha_{2}^{2} k_{2}}{b_{2}+a_{2}^{2}}-\frac{2 \alpha_{1}^{2} a_{2}^{2} k_{1}}{b_{1}+a_{1}^{2}} .
$$

In obtaining the above inequality we have used the fact that $l_{1}$ and $\left(1-l_{2}\right)$ both do not exceed 1. Thus, for $a_{1}, a_{2}$ sufficiently small, it follows that $w=u_{t}=0$ and $z=v_{t}=0$. This establishes uniqueness and completes the proof of Theorem 3.1.

\section{REFERENCES}

[1] K. T. Andrews, A. Mikelić, P. Shi, M. Shillor, and S. Wright, One-dimensional thermoelastic contact with a stress-dependent radiation condition, SIAM J. Math. Anal. 23, 1393-1416 (1992)

[2] K. T. Andrews, P. Shi, M. Shillor, and S. Wright, Thermoelastic contact with Barber's heat exchange condition, Appl. Math. Optim. 28, 11-48 (1993)

[3] K. T. Andrews and M. Shillor, A parabolic initial-boundary value problem modeling axially symmetric thermoelastic contact, Nonlinear Analysis (to appear)

[4] J. R. Barber, Stability of thermoelastic contact, IMech. E. Internat. Conf. Tribology, Mechanical Engineering Publications Ltd., London, 1987, pp. 981-986

[5] J. R. Barber and R. Zhang, Transient behaviour and stability for the thermoelastic contact of two rods of dissimilar materials, Internat. J. Mech. Sci. 30, 691-704 (1988)

[6] D. E. Carlson, Linear thermoelasticity, Handbuch der Physik, Vol. VIa/2 (S. Flugge, ed.), SpringerVerlag, Berlin, 1972, pp. 297-345

[7] C. Cheng and M. Shillor, Numerical solutions to the problem of thermoelastic contact of two rods, Math. and Comput. Modelling 17, no. 10, 53-71 (1993)

[8] M. I. M. Copetti and C. M. Elliott, A one-dimensional quasi-static contact problem in linear thermoelasticity, European J. Appl. Math. 4, 151-174 (1993)

[9] W. A. Day, Heat Conduction with Linear Thermoelasticity, Springer-Verlag, New York, 1985

[10] R. P. Gilbert, P. Shi, and M. Shillor, A quasistatic contact problem in linear thermoelasticity, Rend. Mat. 10, 785-808 (1990)

[11] O. A. Ladyzhenskaya, The Boundary Value Problems of Mathematical Physics, Springer-Verlag, New York, 1985

[12] O. A. Ladyžer skaja, V. A. Solonnikov, and N. N. Ural'ceva, Linear and quasilinear equations of parabolic type, Amer. Math. Soc., Providence, RI, 1968

[13] J. L. Lions and E. Magenes, Nonhomogeneous Boundary Value Problems and Applications, Vol. II, Springer-Verlag, New York, 1972

[14] T. Roubiček, A coupled contact problem in nonlinear thermo-visco-elasticity, preprint

[15] P. Shi and M. Shillor, Uniqueness and stability of the solution to a thermoelastic contact problem, European J. Appl. Math. 1, 371-387 (1990)

[16] P. Shi and M. Shillor, $A$ quasistatic contact problem in thermoelasticity with a radiation condition for the temperature, J. Math. Anal. Appl. 172, 147-165 (1993)

[17] P. Shi and M. Shillor, Existence of a solution to the $N$-dimensional problem of thermoelastic contact, Comm. Partial Differential Equations 17, 1597-1618 (1992)

[18] P. Shi, M. Shillor, and X. L. Zou, Numerical solutions to one-dimensional problems of thermoelastic contact, Comput. Math. Appl. 22, no. 10, 65-78 (1991)

[19] R. Zhang and J. R. Barber, Effect of material properties on the stability of static thermoelastic contact, J. Appl. Mech. 57, 365-369 (1990)

[20] X. Zou, Existence and uniqueness of a solution to the axially symmetric thermoelastic contact problem, M. S. Thesis, Oakland Univ., Rochester, MI, 1991

[21] X. Zou, Existence and uniqueness of a solution to a singular thermoelastic contact problem, Applicable Anal. 51, no. 2, 139 (1993) 